\title{
APPROXIMATION PROPERTIES OF SOME DISCRETE FOURIER SUMS FOR PIECEWISE SMOOTH DISCONTINUOUS FUNCTIONS
}

\begin{abstract}
Denote by $L_{n, N}(f, x)$ a trigonometric polynomial of order at most $n$ possessing the least quadratic deviation from $f$ with respect to the system $\left\{t_{k}=u+\frac{2 \pi k}{N}\right\}_{k=0}^{N-1}$, where $u \in \mathbb{R}$ and $n \leqslant N / 2$. Let $D^{1}$ be the space of $2 \pi$-periodic piecewise continuously differentiable functions $f$ with a finite number of jump discontinuity points $-\pi=\xi_{1}<\ldots<\xi_{m}=\pi$ and with absolutely continuous derivatives on each interval $\left(\xi_{i}, \xi_{i+1}\right)$. In the present article, we consider the problem of approximation of functions $f \in D^{1}$ by the trigonometric polynomials $L_{n, N}(f, x)$. We have found the exact order estimate $\left|f(x)-L_{n, N}(f, x)\right| \leqslant c(f, \varepsilon) / n,\left|x-\xi_{i}\right| \geqslant \varepsilon$. The proofs of these estimations are based on comparing of approximating properties of discrete and continuous finite Fourier series.
\end{abstract}

Key words: function approximation, trigonometric polynomials, Fourier series

\section{Mathematical Subject Classification: 41A25}

1. Introduction. Let $D^{1}$ be the space of $2 \pi$-periodic functions $f$, each of which has a finite number of jump discontinuity points $\Omega(f)=$ $=\left\{\xi_{i}\right\}_{i=0}^{m}$, where $-\pi=\xi_{0}<\xi_{1}<\ldots<\xi_{m}=\pi, f\left(\xi_{i}\right)=\left(f\left(\xi_{i}-0\right)+\right.$ $\left.+f\left(\xi_{i}+0\right)\right) / 2$ and has an absolutely continuous derivative $f^{\prime}$ on each interval $\left(\xi_{i}, \xi_{i+1}\right)(0 \leqslant i \leqslant m)$ (here we say that a function $f$ is absolutely continuous on an interval $(a, b)$ if the function $\bar{f}$ is absolutely continuous on the segment $[a, b]$, where $\bar{f}(x)=f(x)$ for $x \in(a, b), \bar{f}(a)=f(a+0)$, and $\bar{f}(b)=f(b-0))$. One of such functions is $f(x)=\operatorname{sign}(\sin x)$.

Denote by $L_{n, N}(f, x)(1 \leqslant n \leqslant\lfloor N / 2\rfloor)$ the trigonometric polynomial of order at most $n$ that possesses the least quadratic deviation from the function $f$ with respect to the system $\left\{t_{k}\right\}_{k=0}^{N-1}$, where $t_{k}=u+2 \pi k / N$ $(u \in \mathbb{R})$. In other words, the minimum of the sums $\sum_{k=0}^{N-1}\left|f\left(t_{k}\right)-T_{n}\left(t_{k}\right)\right|^{2}$

(C) Petrozavodsk State University, 2019 
on the set of trigonometric polynomials $T_{n}$ of order $n$ is attained when $T_{n}=L_{n, N}(f)$. In particular, $L_{\lfloor N / 2\rfloor, N}\left(f, t_{k}\right)=f\left(t_{k}\right)$. It is easy to show (see [13]) that for $n<N / 2$ the polynomial $L_{n, N}(f, x)$ can be represented as follows:

$$
L_{n, N}(f, x)=\sum_{\nu=-n}^{n} c_{\nu}^{(N)}(f) e^{i \nu x}, \quad c_{\nu}^{(N)}(f)=\frac{1}{N} \sum_{k=0}^{N-1} f\left(t_{k}\right) e^{-i \nu t_{k}}
$$

and for $n=N / 2$ :

$$
L_{N / 2, N}(f, x)=L_{N / 2-1, N}(f, x)+a_{N / 2}^{(N)}(f) \cos \frac{N}{2}(x-u),
$$

where

$$
a_{n}^{(2 n)}(f)=a_{N / 2}^{(N)}(f)=\frac{1}{N} \sum_{k=0}^{N-1} f\left(t_{k}\right) \cos \frac{N}{2}\left(t_{k}-u\right) .
$$

By $S_{n}(f, x)$ we denote the partial Fourier sum of order $n$ of $f$ :

$$
S_{n}(f, x)=\frac{a_{0}}{2}+\sum_{k=1}^{n}\left(a_{k} \cos k x+b_{k} \sin k x\right),
$$

where

$$
a_{k}=\frac{1}{\pi} \int_{-\pi}^{\pi} f(t) \cos k t d t, \quad b_{k}=\frac{1}{\pi} \int_{-\pi}^{\pi} f(t) \sin k t d t .
$$

To read more about approximation of functions by trigonometric polynomials, see [4-7], [9-12], [14].

Also, later we will need the function

$$
h_{p}(x)= \begin{cases}\cos x, & p=0 \\ \sin x, & p=1\end{cases}
$$

and the well-known inequalities

$$
\begin{gathered}
\left|\sum_{k=1}^{\infty} \frac{\sin k x}{k}\right| \leqslant \frac{\pi}{2}, \\
\left|\sum_{k=1}^{n} h_{p}(k x)\right| \leqslant \frac{1}{\left|\sin \frac{x}{2}\right|}, \quad x \neq 2 i \pi, \quad i=0, \pm 1, \pm 2, \ldots,
\end{gathered}
$$




$$
\sum_{k=1}^{\infty} \frac{1}{k^{2}}=\frac{\pi^{2}}{6} .
$$

It is easy to show, that the Fourier series converges pointwise for any function $f \in D^{1}$ and, therefore, the function can be represented as follows:

$$
f(x)=\frac{a_{0}}{2}+\sum_{k=1}^{\infty}\left(a_{k} \cos k x+b_{k} \sin k x\right) .
$$

In the previous works, the author found estimates for the value $\left|f(x)-L_{n, N}(f, x)\right|$ for $2 \pi$-periodic piecewise-linear and piecewise-smooth continuous functions (see [1], [2]). Also, two particular cases of such functions $-2 \pi$-periodic functions $f(x)=|x|$ and $f(x)=\operatorname{sign} x$, $x \in[-\pi, \pi]$ - were considered in [3]. The goal of this work is to estimate $\left|f(x)-L_{n, N}(f, x)\right|$ for $f \in D^{1}$ as $n, N \rightarrow \infty$. We obtained the following result:

Theorem 1. For a function $f \in D^{1}$, the following estimate holds:

$$
\left|f(x)-L_{n, N}(f, x)\right| \leqslant \frac{C(f, \varepsilon)}{n}, \quad 1 \leqslant n \leqslant\lfloor N / 2\rfloor, \quad\left|x-\xi_{i}\right|>\varepsilon,
$$

where $i=0,1, \ldots, m$. The order of this estimate cannot be improved.

To prove this theorem, we use a lemma from [13]:

Lemma 1. [13] If the Fourier series of $f$ converges at the points $t_{k}=u+2 k \pi / N$, then the representation

$$
L_{n, N}(f, x)=S_{n}(f, x)+R_{n, N}(f, x),
$$

where

$$
\begin{gathered}
R_{n, N}(f, x)=\frac{2}{\pi} \sum_{\mu=1}^{\infty} \int_{-\pi}^{\pi} f(t) D_{n}(x-t) \cos \mu N(u-t) d t \\
D_{n}(x)=\frac{1}{2}+\sum_{k=1}^{n} \cos k x
\end{gathered}
$$

holds true when $2 n<N$.

From this lemma, we have the following estimate:

$$
\left|f(x)-L_{n, N}(f, x)\right| \leqslant\left|f(x)-S_{n}(f, x)\right|+\left|R_{n, N}(f, x)\right|, \quad n<N / 2 .
$$


In the case $2 n=N$, from (1) and (9) we have

$$
\begin{aligned}
& \left|f(x)-L_{n, N}(f, x)\right| \leqslant \\
& \quad \leqslant\left|f(x)-S_{n-1}(f, x)\right|+\left|R_{n-1, N}(f, x)\right|+\left|a_{n}^{(N)}(f)\right|, \quad n=N / 2 .
\end{aligned}
$$

The estimate for $\left|f(x)-S_{n}(f, x)\right|$, where $f \in D^{1}$, were obtained in [8]:

$$
\left|f(x)-S_{n}(f, x)\right| \leqslant \frac{C(f, \varepsilon)}{n}, \quad\left|x-\xi_{i}\right| \geqslant \varepsilon .
$$

Now we have to estimate the values $\left|R_{n, N}(f, x)\right|$ and $\left|a_{n}^{(2 n)}(f)\right|$, which is done in the following sections.

2. The estimate for $\left|R_{n, N}(f, x)\right|$. From (7) and (8), we can get the representation

$$
\begin{aligned}
R_{n, N}(f, x) & =\frac{1}{\pi} \sum_{\mu=1}^{\infty} \int_{-\pi}^{\pi} f(t) \cos \mu N(u-t) d t+ \\
+\frac{2}{\pi} \sum_{\mu=1}^{\infty} \int_{-\pi}^{\pi} f(t) \sum_{k=1}^{n} \cos k(x-t) \cos \mu N(u-t) d t & \\
& =R_{n, N}^{1}(f, x)+R_{n, N}^{2}(f, x) .
\end{aligned}
$$

Lemma 2. For $\alpha \in\left(0, \frac{1}{2}\right]$, the following inequality holds:

$$
\left|\sum_{k=1}^{\infty} \frac{\sin k x}{k\left(1-\frac{\alpha^{2}}{k^{2}}\right)}\right| \leqslant c .
$$

Proof. Performing the Abel transformation (summation by parts), we get

$$
\begin{aligned}
\sum_{k=1}^{\infty} \frac{\sin k x}{k\left(1-\frac{\alpha^{2}}{k^{2}}\right)} & =\sum_{k=1}^{\infty}\left(\frac{1}{1-\frac{\alpha^{2}}{k^{2}}}-\frac{1}{1-\frac{\alpha^{2}}{(k+1)^{2}}}\right) \sum_{j=1}^{k} \frac{\sin j x}{j}= \\
& =\sum_{k=1}^{\infty} \frac{1}{k^{2}} \frac{\alpha^{2}\left(2+\frac{1}{k}\right)}{\left(1+\frac{1}{k}\right)^{2}\left(1-\frac{\alpha^{2}}{k^{2}}\right)\left(1-\frac{\alpha^{2}}{(k+1)^{2}}\right)} \frac{1}{k} \sum_{j=1}^{k} \frac{\sin j x}{j}
\end{aligned}
$$

Using (5) and the inequalities

$$
\frac{\alpha^{2}\left(2+\frac{1}{k}\right)}{\left(1+\frac{1}{k}\right)^{2}\left(1-\frac{\alpha^{2}}{k^{2}}\right)\left(1-\frac{\alpha^{2}}{(k+1)^{2}}\right)} \leqslant \frac{16}{15}, \quad\left|\frac{1}{k} \sum_{j=1}^{k} \frac{\sin j x}{j}\right| \leqslant 1
$$


we have

$$
\begin{aligned}
& \left|\sum_{k=1}^{\infty} \frac{\sin k x}{k\left(1-\frac{\alpha^{2}}{k^{2}}\right)}\right| \leqslant \\
& \leqslant \sum_{k=1}^{\infty} \frac{1}{k^{2}} \frac{\alpha^{2}\left(2+\frac{1}{k}\right)}{\left(1+\frac{1}{k}\right)^{2}\left(1-\frac{\alpha^{2}}{k^{2}}\right)\left(1-\frac{\alpha^{2}}{(k+1)^{2}}\right)}\left|\frac{1}{k} \sum_{j=1}^{k} \frac{\sin j x}{j}\right| \leqslant c .
\end{aligned}
$$

This completes the proof.

Lemma 3. For $f \in D^{1}$, the following holds:

$$
\begin{aligned}
& \int_{-\pi}^{\pi} f(t) h_{p}(k(t-x)) h_{q}(\mu N(t-u)) d t= \\
& =\frac{(-1)^{q} \mu N}{(\mu N)^{2}-k^{2}} \sum_{i=0}^{m-1}\left(f\left(\xi_{i}-0\right)-f\left(\xi_{i}+0\right)\right) h_{p}\left(k\left(\xi_{i}-x\right)\right) h_{1-q}\left(\mu N\left(\xi_{i}-u\right)\right)- \\
& \quad-\frac{(-1)^{q} \mu N}{(\mu N)^{2}-k^{2}} \int_{-\pi}^{\pi} f^{\prime}(t) h_{p}(k(t-x)) h_{1-q}(\mu N(t-u)) d t+ \\
& +\frac{(-1)^{1+p} k}{(\mu N)^{2}-k^{2}} \sum_{i=0}^{m-1}\left(f\left(\xi_{i}-0\right)-f\left(\xi_{i}+0\right)\right) h_{1-p}\left(k\left(\xi_{i}-x\right)\right) h_{q}\left(\mu N\left(\xi_{i}-u\right)\right)- \\
& -\frac{(-1)^{1+p} k}{(\mu N)^{2}-k^{2}} \int_{-\pi}^{\pi} f^{\prime}(t) h_{1-p}(k(t-x)) h_{q}(\mu N(t-u)) d t . \quad(12)
\end{aligned}
$$

Proof. Perform integration by parts:

$$
\begin{aligned}
& \int_{-\pi}^{\pi} f(t) h_{p}(k(t-x)) h_{q}(\mu N(t-u)) d t= \\
& =\frac{(-1)^{q}}{\mu N} \sum_{i=0}^{m-1}\left(f\left(\xi_{i}-0\right)-f\left(\xi_{i}+0\right)\right) h_{p}\left(k\left(\xi_{i}-x\right)\right) h_{1-q}\left(\mu N\left(\xi_{i}-u\right)\right)- \\
& \quad-\frac{(-1)^{q}}{\mu N} \int_{-\pi}^{\pi} f^{\prime}(t) h_{p}(k(t-x)) h_{1-q}(\mu N(t-u)) d t+
\end{aligned}
$$




$$
+\frac{(-1)^{p+q} k}{\mu N} \int_{-\pi}^{\pi} f(t) h_{1-p}(k(t-x)) h_{1-q}(\mu N(t-u)) d t
$$

Repeat integration by parts for the last integral in (13):

$$
\begin{aligned}
& \int_{-\pi}^{\pi} f(t) h_{p}(k(t-x)) h_{q}(\mu N(t-u)) d t= \\
& =\frac{(-1)^{q}}{\mu N} \sum_{i=0}^{m-1}\left(f\left(\xi_{i}-0\right)-f\left(\xi_{i}+0\right)\right) h_{p}\left(k\left(\xi_{i}-x\right)\right) h_{1-q}\left(\mu N\left(\xi_{i}-u\right)\right)- \\
& \quad-\frac{(-1)^{q}}{\mu N} \int_{-\pi}^{\pi} f^{\prime}(t) h_{p}(k(t-x)) h_{1-q}(\mu N(t-u)) d t+ \\
& +\frac{(-1)^{1+p} k}{(\mu N)^{2}} \sum_{i=0}^{m-1}\left(f\left(\xi_{i}-0\right)-f\left(\xi_{i}+0\right)\right) h_{1-p}\left(k\left(\xi_{i}-x\right)\right) h_{q}\left(\mu N\left(\xi_{i}-u\right)\right)- \\
& -\frac{(-1)^{1+p} k}{(\mu N)^{2}} \int_{-\pi}^{\pi} f^{\prime}(t) h_{1-p}(k(t-x)) h_{q}(\mu N(t-u)) d t+ \\
& +\frac{k^{2}}{(\mu N)^{2}} \int_{-\pi}^{\pi} f(t) h_{p}(k(t-x)) h_{q}(\mu N(t-u)) d t
\end{aligned}
$$

By moving the last integral to the left-hand side and dividing both sides by $\frac{(\mu N)^{2}-k^{2}}{(\mu N)^{2}}$, we get $(12)$.

Lemma 4. The value $\left|R_{n, N}^{1}(f, x)\right|$, where $f \in D^{1}$, can be estimated as follows:

$$
\left|R_{n, N}^{1}(f, x)\right| \leqslant \frac{c(f)}{N} .
$$

Proof. Performing integration by parts twice, we get

$$
\begin{aligned}
& R_{n, N}^{1}(f, x)= \\
& =\frac{1}{\pi} \sum_{\mu=1}^{\infty} \int_{-\pi}^{\pi} f(t) \cos \mu N(t-u) d t=\frac{1}{\pi} \sum_{\mu=1}^{\infty} \sum_{i=0}^{m-1} \int_{\xi_{i}}^{\xi_{i+1}} f(t) \cos \mu N(t-u) d t=
\end{aligned}
$$




$$
\begin{gathered}
=\frac{1}{\pi N} \sum_{\mu=1}^{\infty} \frac{1}{\mu} \sum_{i=0}^{m-1}\left(f\left(\xi_{i}-0\right)-f\left(\xi_{i}+0\right)\right) \sin \mu N\left(\xi_{i}-u\right)+ \\
+\frac{1}{\pi N^{2}} \sum_{\mu=1}^{\infty} \frac{1}{\mu^{2}}\left[\sum_{i=0}^{m-1}\left(f^{\prime}\left(\xi_{i}-0\right)-f^{\prime}\left(\xi_{i}+0\right)\right) \cos \mu N\left(\xi_{i}-u\right)-\right. \\
\left.-\int_{-\pi}^{\pi} f^{\prime \prime}(t) \cos \mu N(t-u) d t\right]
\end{gathered}
$$

Applying some simple transformations and using (3), we have

$$
\begin{aligned}
& \left|R_{n, N}^{1}(f, x)\right| \leqslant \frac{1}{\pi N} \sum_{i=0}^{m-1}\left|f\left(\xi_{i}-0\right)-f\left(\xi_{i}+0\right)\right|\left|\sum_{\mu=1}^{\infty} \frac{\sin \mu N\left(\xi_{i}-u\right)}{\mu}\right|+ \\
& +\frac{1}{\pi N^{2}} \sum_{\mu=1}^{\infty} \frac{1}{\mu^{2}}\left[\sum_{i=0}^{m-1}\left|f^{\prime}\left(\xi_{i}-0\right)-f^{\prime}\left(\xi_{i}+0\right)\right|+\int_{-\pi}^{\pi}\left|f^{\prime \prime}(t)\right| d t\right] \leqslant \frac{c(f)}{N} .
\end{aligned}
$$

This completes the proof.

Lemma 5. The value $\left|R_{n, N}^{2}(f, x)\right|$, where $f \in D^{1}$, can be estimated as follows:

$$
\left|R_{n, N}^{2}(f, x)\right| \leqslant \frac{c(f, \varepsilon)}{N}, \quad\left|x-\xi_{i}\right| \geqslant \varepsilon .
$$

Proof. Using Lemma 3, we have

$$
\begin{aligned}
& R_{n, N}^{2}(f, x)=\frac{2}{\pi} \sum_{\mu=1}^{\infty} \sum_{k=1}^{n} \int_{-\pi}^{\pi} f(t) \cos k(t-x) \cos \mu N(t-u) d t= \\
& =\frac{2}{\pi N} \sum_{i=0}^{m-1}\left(f\left(\xi_{i}-0\right)-f\left(\xi_{i}+0\right)\right) \sum_{\mu=1}^{\infty} \frac{\sin \mu N\left(\xi_{i}-u\right)}{\mu} \sum_{k=1}^{n} \frac{\cos k\left(\xi_{i}-x\right)}{1-\left(\frac{k}{\mu N}\right)^{2}}+ \\
& \quad+\frac{-2}{\pi N} \sum_{\mu=1}^{\infty} \frac{1}{\mu} \sum_{k=1}^{n} \frac{1}{1-\left(\frac{k}{\mu N}\right)^{2}} \int_{-\pi}^{\pi} f^{\prime}(t) \cos k(t-x) \sin \mu N(t-u) d t+ \\
& +\frac{-2}{\pi N^{2}} \sum_{i=0}^{m-1}\left(f\left(\xi_{i}-0\right)-f\left(\xi_{i}+0\right)\right) \sum_{\mu=1}^{\infty} \frac{\cos \mu N\left(\xi_{i}-u\right)}{\mu^{2}} \sum_{k=1}^{n} \frac{k \sin k\left(\xi_{i}-x\right)}{1-\left(\frac{k}{\mu N}\right)^{2}}+
\end{aligned}
$$




$$
\begin{array}{r}
+\frac{2}{\pi N^{2}} \sum_{\mu=1}^{\infty} \frac{1}{\mu^{2}} \sum_{k=1}^{n} \frac{k}{1-\left(\frac{k}{\mu N}\right)^{2}} \int_{-\pi}^{\pi} f^{\prime}(t) \sin k(t-x) \cos \mu N(t-u) d t= \\
=R_{n, N}^{2.1}(f, x)+R_{n, N}^{2.2}(f, x)+R_{n, N}^{2.3}(f, x)+R_{n, N}^{2.4}(f, x)
\end{array}
$$

Here we estimate only the values $\left|R_{n, N}^{2.1}(f, x)\right|$ and $\left|R_{n, N}^{2.2}(f, x)\right|$, because $\left|R_{n, N}^{2.3}(f, x)\right|$ and $\left|R_{n, N}^{2.4}(f, x)\right|$ can be estimated in the similar way. Begin with $\left|R_{n, N}^{2.1}(f, x)\right|$. Consider the expression

$$
A=\sum_{k=1}^{n} \cos k\left(\xi_{i}-x\right) \sum_{\mu=1}^{\infty} \frac{\sin \mu N\left(\xi_{i}-u\right)}{\mu\left(1-\left(\frac{k}{\mu N}\right)^{2}\right)} .
$$

Applying the Abel transformation, we get

$$
\begin{aligned}
& A=\sum_{\mu=1}^{\infty} \frac{\sin \mu N\left(\xi_{i}-u\right)}{\mu\left(1-\left(\frac{n}{\mu N}\right)^{2}\right)} \sum_{j=1}^{n} \cos j\left(\xi_{i}-x\right)+ \\
& +\sum_{k=1}^{n-1} \sum_{\mu=1}^{\infty} \frac{\sin \mu N\left(\xi_{i}-u\right)}{\mu}\left(\frac{1}{1-\left(\frac{k}{\mu N}\right)^{2}}-\frac{1}{1-\left(\frac{k+1}{\mu N}\right)^{2}}\right) \sum_{j=1}^{k} \cos j\left(\xi_{i}-x\right) .
\end{aligned}
$$

Using (4), Lemma 2 and the fact that

$$
\frac{1}{1-\left(\frac{k}{\mu N}\right)^{2}}-\frac{1}{1-\left(\frac{k+1}{\mu N}\right)^{2}}=-\frac{k}{(\mu N)^{2}} \frac{2+\frac{1}{k}}{\left(1-\left(\frac{k}{\mu N}\right)^{2}\right)\left(1-\left(\frac{k+1}{\mu N}\right)^{2}\right)}
$$

we get

$$
|A| \leqslant \frac{c}{\left|\sin \frac{\xi_{i}-x}{2}\right|} .
$$

From this, we get the estimate for $\left|R_{n, N}^{2.1}(f, x)\right|$ :

$$
\begin{aligned}
& \left|R_{n, N}^{2.1}(f, x)\right| \leqslant \\
& \frac{c}{N} \sum_{i=0}^{m-1}\left|\frac{f\left(\xi_{i}-0\right)-f\left(\xi_{i}+0\right)}{\sin \frac{\xi_{i}-x}{2}}\right| \leqslant \frac{c(f, \varepsilon)}{N}, \quad\left|x-\xi_{i}\right| \geqslant \varepsilon .
\end{aligned}
$$


In the similar way, we get the estimate

$$
\left|R_{n, N}^{2.3}(f, x)\right| \leqslant \frac{c(f, \varepsilon)}{N}, \quad\left|x-\xi_{i}\right| \geqslant \varepsilon .
$$

Now we estimate $\left|R_{n, N}^{2.2}(f, x)\right|$. Consider the integral

$$
B=\int_{-\pi}^{\pi} f^{\prime}(t) \cos k(t-x) \sin \mu N(t-u) d t .
$$

Using Lemma 3, we estimate the value $|B|$ as follows:

$$
|B| \leqslant \frac{c}{\mu N}\left[\sum_{i=0}^{m-1}\left|f^{\prime}\left(\xi_{i}-0\right)-f^{\prime}\left(\xi_{i}+0\right)\right|+\int_{-\pi}^{\pi}\left|f^{\prime \prime}(t)\right| d t\right] \leqslant \frac{c(f)}{\mu N} .
$$

Now we have

$$
\left|R_{n, N}^{2.2}(f, x)\right|=\left|\frac{2}{\pi N} \sum_{\mu=1}^{\infty} \frac{1}{\mu} \sum_{k=1}^{n} \frac{B}{1-\left(\frac{k}{\mu N}\right)^{2}}\right| \leqslant \frac{c(f)}{N} .
$$

The value $\left|R_{n, N}^{2.4}(f, x)\right|$ can be estimated in the similar way:

$$
\left|R_{n, N}^{2.4}(f, x)\right| \leqslant \frac{c(f)}{N}
$$

From (14)-(17) we have

$$
\left|R_{n, N}^{2}(f, x)\right| \leqslant \frac{c(f, \varepsilon)}{N}, \quad\left|x-\xi_{i}\right| \geqslant \varepsilon .
$$

Lemma is proved.

Finally, from Lemmas 4 and 5, we have

$$
\left|R_{n, N}(f, x)\right| \leqslant \frac{c(f, \varepsilon)}{N}, \quad\left|x-\xi_{i}\right| \geqslant \varepsilon .
$$

3. The estimate for $\left|a_{n}^{(2 n)}(f)\right|$. From (2), using that $t_{j}=u+2 \pi k / N$, we have

$$
a_{n}^{(N)}(f)=\frac{1}{N} \sum_{k=0}^{2 n-1}(-1)^{k} f\left(t_{k}\right)=\frac{1}{N} \sum_{k=0}^{n-1}\left(f\left(t_{2 k}\right)-f\left(t_{2 k+1}\right)\right)
$$


and

$$
\left|a_{n}^{N}(f)\right| \leqslant \frac{1}{N} \sum_{k=0}^{n-1}\left|f\left(t_{2 k}\right)-f\left(t_{2 k+1}\right)\right| .
$$

Denote by $G$ the subset of indexes $\{k\}_{k=0}^{n-1}$, such that for $k \in G$ the segment $\left[t_{2 k}, t_{2 k+1}\right]$ does not contain any point $\xi_{i}, 0 \leqslant i \leqslant m$. Denote $\hat{G}=\{k\}_{k=0}^{n-1} \backslash G$. Now write

$$
\left|a_{n}^{N}(f)\right| \leqslant \frac{1}{N} \sum_{k \in G}\left|f\left(t_{2 k}\right)-f\left(t_{2 k+1}\right)\right|+\frac{1}{N} \sum_{k \in \hat{G}}\left|f\left(t_{2 k}\right)-f\left(t_{2 k+1}\right)\right| .
$$

For each $k \in G$, the segment $\left[t_{2 k}, t_{2 k+1}\right]$ lies entirely inside some interval $\left(\xi_{i}, \xi_{i+1}\right)$ and, therefore, the function $f$ is differentiable on it, which allows us to use the mean-value theorem and get the following inequality:

$$
\left|f\left(t_{2 k}\right)-f\left(t_{2 k+1}\right)\right| \leqslant c(f)\left|t_{2 k}-t_{2 k+1}\right| \leqslant \frac{c(f)}{N} .
$$

For a $k \in \hat{G}$, there are $s(k)$ points $\xi_{i_{k, 1}}<\xi_{i_{k, 2}}<\ldots<\xi_{i_{k, s}(k)}$ inside the segment $\left[t_{2 k}, t_{2 k+1}\right]$. Now we estimate the value $\left|f\left(t_{2 k}\right)-f\left(t_{2 k+1}\right)\right|$ for $k \in \hat{G}$. First, we need the following lemma:

Lemma 6. For $f \in D^{1}$ and the segment $[a, b]$, where $[a, b] \subset[-\pi, \pi]$, the following holds:

$$
|f(a)-f(b)| \leqslant c(f)(s+|a-b|),
$$

where $s$ is the number of jump discontinuity points $x_{1}, x_{1}, \ldots, x_{s}$ of the function $f$ on the segment $[a, b]$.

Proof. Here we consider only the case $a<x_{i}<\ldots<x_{s}<b$. The proof for the cases $a=x_{1}$ or $b=x_{s}$ is similar. Consider the following inequality:

$$
\begin{aligned}
|f(a)-f(b)| \leqslant & \left|f(a)-f\left(x_{1}-0\right)\right|+\sum_{i=1}^{s}\left|f\left(x_{i}-0\right)-f\left(x_{i}+0\right)\right|+ \\
& +\sum_{i=1}^{s-1}\left|f\left(x_{i}+0\right)-f\left(x_{i+1}-0\right)\right|+\left|f\left(x_{s}+0\right)-f(b)\right| .
\end{aligned}
$$

Function $f$ is differentiable on each of the intervals $\left(a, x_{1}\right),\left(x_{1}, x_{2}\right), \ldots$, $\left(x_{s-1}, x_{s}\right),\left(x_{s}, b\right)$. Using the mean-value theorem, we can write 


$$
\begin{aligned}
|f(a)-f(b)| \leqslant c(f)|a-b| & +\sum_{i=1}^{s}\left|f\left(x_{i}-0\right)-f\left(x_{i}+0\right)\right| \leqslant \\
& \leqslant c(f)|a-b|+s M \leqslant c(f)|a-b|+c(f) s
\end{aligned}
$$

where $M=\max _{1 \leqslant i \leqslant s}\left|f^{\prime}\left(x_{i}-0\right)-f^{\prime}\left(x_{i}+0\right)\right|$.

From this lemma

$$
\begin{aligned}
\sum_{k \in \hat{G}}\left|f\left(t_{2 k}\right)-f\left(t_{2 k+1}\right)\right| & \\
& \leqslant \sum_{k \in \hat{G}} c(f)\left(s(k)+\frac{2 \pi}{N}\right) \leqslant c(f) \sum_{k \in \hat{G}} s(k)+\sum_{k \in \hat{G}} \frac{2 \pi}{N} .
\end{aligned}
$$

Each point $\xi_{1}, \xi_{2}, \ldots, \xi_{m-1}$ may be included in one or two segments $\left[t_{2 k}, t_{2 k+1}\right], k \in \hat{G}$, therefore, $\sum_{k \in \hat{G}} s(k)<2 m$. Using this and the fact that $|\hat{G}| \leqslant m$, we have

$$
\sum_{k \in \hat{G}}\left|f\left(t_{2 k}\right)-f\left(t_{2 k+1}\right)\right| \leqslant c(f)
$$

From (19), (20), and (21) inequality

$$
\left|a_{n}^{N}(f)\right| \leqslant \frac{c(f)}{N}
$$

follows.

4. The proof of Theorem 1 . The proof of estimate (6) from Theorem 1 immediately follows from inequalities (9), (10), (11), (18), (22), and $n \leqslant N / 2$. To prove that the order of this estimate cannot be improved, consider the value $\left|f_{1}\left(\frac{\pi}{2}\right)-L_{4 n, N}\left(f_{1}, \frac{\pi}{2}\right)\right|$, where $4 n<N / 2$ and $f_{1}(x)=$ $=\operatorname{sign}(\sin x)$. From Lemma 1 , get the inequality

$$
\left|f(x)-L_{n, N}(f, x)\right| \geqslant\left|f(x)-S_{n}(f, x)\right|-\left|R_{n, N}(f, x)\right| .
$$

It is easy to show that the following representation takes place:

$$
f_{1}(x)=\frac{2}{\pi} \sum_{k=1}^{\infty} \frac{\left(1-(-1)^{k}\right) \sin k x}{k}=\frac{4}{\pi} \sum_{k=1}^{\infty} \frac{\sin (2 k-1) \pi}{2 k-1}
$$




$$
S_{2 n}\left(f_{1}, x\right)=\frac{4}{\pi} \sum_{k=1}^{n} \frac{\sin (2 k-1) x}{2 k-1} .
$$

Using this, we can estimate the value $\left|f_{1}\left(\frac{\pi}{2}\right)-S_{4 n}\left(f_{1}, \frac{\pi}{2}\right)\right|$ from below:

$$
\begin{aligned}
\left|f_{1}\left(\frac{\pi}{2}\right)-S_{4 n}\left(f_{1}, \frac{\pi}{2}\right)\right|= & \\
=\frac{4}{\pi}\left|\sum_{k=2 n+1}^{\infty} \frac{(-1)^{k+1}}{2 k-1}\right|= & \frac{4}{\pi} \sum_{k=n+1}^{\infty}\left(\frac{1}{4 k-3}-\frac{1}{4 k-1}\right)= \\
= & \frac{8}{\pi} \sum_{k=n+1}^{\infty} \frac{1}{k^{2}\left(4-\frac{1}{k}\right)\left(4-\frac{3}{k}\right)}>\frac{1 / 4}{4 n}
\end{aligned}
$$

From this and (23) we have

$$
\left|f_{1}\left(\frac{\pi}{2}\right)-L_{4 n, N}\left(f_{1}, \frac{\pi}{2}\right)\right| \geqslant \frac{1 / 4}{4 n}-\left|R_{4 n, N}\left(f_{1}, \frac{\pi}{2}\right)\right| .
$$

In the previous sections we showed that $\left|R_{4 n, N}\left(f_{1}, \frac{\pi}{2}\right)\right| \leqslant c / N$. Denote by $N(n)$ a number such that for each $N \geqslant N(n)$ inequality $\left|R_{4 n, N}\left(f_{1}, \frac{\pi}{2}\right)\right| \leqslant \frac{1 / 8}{4 n}$ holds. Now, we have

$$
\left|f_{1}\left(\frac{\pi}{2}\right)-L_{4 n, N(n)}\left(f_{1}, \frac{\pi}{2}\right)\right| \geqslant \frac{1 / 8}{4 n}=\frac{c}{4 n} .
$$

From this we see that the order of estimate (6) cannot be improved. Theorem 1 is proved.

\section{References}

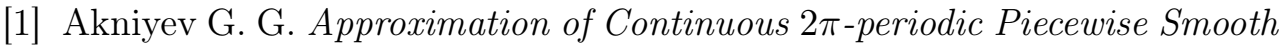
Functions by Discrete Fourier Sums // Izv. Saratov Univ. (N. S.), Ser. Math. Mech. Inform., 2019, vol. 19, iss. 1, pp. 4-15.

DOI: https://doi.org/10.18500/1816-9791-2018-18-4-4-15

[2] Akniyev G. G. Approximation Properties of Discrete Fourier Sums for Some Piecewise Linear Functions // Izv. Saratov Univ. (N. S.), Ser. Math. Mech. Inform., 2018, vol. 18, iss. 1, pp. 4-16 (in Russian).

DOI: https://doi.org/10.18500/1816-9791-2018-18-1-4-16

[3] Akniyev G. G. Discrete least squares approximation of piecewise-linear functions by trigonometric polynomials. Probl. Anal. Issues Anal., 2017, vol. $6(24)$, no. 2 , pp. $3-24$.

DOI: https://doi.org/10.15393/j3.art.2017.4070 
[4] Bernshtein S. N. On trigonometric interpolation by the method of least squares. Dokl. Akad. Nauk USSR, 1934, vol. 4, pp. 1-5 (in Russian).

[5] Erdös P. Some theorems and remarks on interpolation. Acta Sci. Math., Szeged, 1950, vol. 12, pp. $11-17$.

[6] Kalashnikov M. D. On polynomials of best (quadratic) approximation on a given system of points. Dokl. Akad. Nauk USSR, 1955, vol. 105, pp. 634636 (in Russian).

[7] Krilov V. I. Convergence of algebraic interpolation with respect to the roots of a Chebyshev polynomial for absolutely continuous functions and functions with bounded variation. Dokl. Akad. Nauk USSR, 1956, vol. 107, pp. $362-365$ (in Russian).

[8] Magomed-Kasumov M. G. Approximation of piecewise smooth functions by the trigonometric Fourier series. Materials of XIX International Saratov Winter School «Contemporary Problems of Function Theory and Their Applications», 2018, pp. 190-193 (in Russian).

[9] Marcinkiewicz J. Quelques remarques sur l'interpolation. Acta Sci. Math. (Szeged), 1936, vol. 8, pp. 127-130 (in French).

[10] Marcinkiewicz J. Sur la divergence des polynômes d'interpolation. Acta Sci. Math. (Szeged), 1936, vol. 8, pp. 131-135 (in French).

[11] Natanson I. P. On the Convergence of Trigonometrical Interpolation at Equi-Distant Knots. Annals of Mathematics, Second Series, 1944, vol. 45, no. 3, pp. 457 -471. DOI: https ://doi.org/10.2307/1969188

[12] Nikol'skii S. M. On some methods of approximation by trigonometric sums. Mathematics of the USSR - Izvestiya, 1940, vol. 4, pp. 509-520 (in Russian).

[13] Sharapudinov I. I. On the best approximation and polynomials of the least quadratic deviation. Anal. Math., 1983, vol. 9, issue 3, pp. 223-234.

DOI: https ://doi.org/10.7868/S0869565214260041

[14] Zygmund A. Trigonometric Series, vol. 1, Cambridge. : Cambridge University Press, 1959. $747 \mathrm{p}$.

Received November 21, 2018.

In revised form, September 24, 2019.

Accepted September 24, 2019.

Published online October 15, 2019.

Dagestan Federal Research Center of the Russian Academy of Sciences

45 Gadzhieva st., Makhachkala 367025, Russia

E-mail: hasan.akniyev@gmail.com 